\section{Aromatase inhibitor therapy in a cystic fibrosis patient with thoracic endometriosis}

\author{
Olga T. Filippova, ${ }^{1}$ Heidi E. Godoy, ${ }^{1,2}$ \\ Patrick F. Timmins III,2 \\ ${ }^{1}$ Albany Medical Center, Albany, NY; \\ ${ }^{2}$ Women's Cancer Care Associates, \\ Albany, NY, USA
}

\section{Abstract}

The thoracic cavity is the number one site for extrapelvic endometriosis, with catamenial pneumothorax as the most common presenting symptom. Its treatment algorithm is similar to the one for pelvic endometriosis, with the goal of inducing a hypoestrogenic state. However, if medical treatment fails, lung resection may be the only option. We present a case of a 44-year-old female with cystic fibrosis and known pelvic endometriosis, who was diagnosed with thoracic endometriosis after presenting with catamenial hemoptysis. After having a recurrence more than four years after a bilateral salpingo-oophorectomy, she was started on aromatase inhibitor (AI) therapy to avoid lung resection. Such therapy with an AI successfully treated recurrent thoracic endometriosis in a patient with cystic fibrosis, i.e. not an ideal candidate for lung resection.

\section{Introduction}

Endometriosis - the presence of endometrial glands and stroma outside of the uterine cavity - has been described in nearly every site, including the umbilicus, scars, breasts, extremities, and the pleural cavity (the most common location). ${ }^{1,2}$ Extrapelvic endometriosis occurs in $14-26 \%$ of women with pelvic endometriosis, yet pelvic endometriosis is documented in $50-84 \%$ of patients with thoracic endometriosis syndrome (TES). ${ }^{1}$ The mean age of presentation of TES is 34.2 years. ${ }^{1}$

Thoracic endometriosis was first described by Maurer and colleagues in $1958 .{ }^{3}$ Presenting symptoms include catamenial pneumothorax (73-80\%), catamenial hemothorax (14\%), catamenial hemoptysis (5-7\%), and lung nodules $(6 \%))^{1,2}$ Up to one third of women with a diagnosis of spontaneous pneumothorax actually have a catamenial pneumothorax, which is defined as a recurrent pneumothorax occurring $24 \mathrm{~h}$ before or up to $72 \mathrm{~h}$ after the onset of menstruation. ${ }^{1}$ A high level of suspicion is needed for diagnosis, as two thirds of the diagnoses are established on historical criteria alone, and can be delayed until the temporal relationship between symptoms and menses is recognised. ${ }^{2}$ The hemoptysis is rarely lifethreatening, but there has been a report of cystic fibrosis (CF) patients having life-threatening hemoptysis, which coincided with infectious exacerbations of $\mathrm{CF}^{4}$

Much of management of TES is drawn from case reports and small clinical series, but still revolves around medical versus surgical treatment. Initially, it is treated with the same agents as pelvic endometriosis-combined contraceptives, progestins, gonadotropin releasing hormone (GnRH) analogues, and clomiphene citrate. ${ }^{1,2}$ A hysterectomy and bilateral salpingo-oophorectomy becomes an option once medical management has failed, the patient cannot tolerate the side effects, or symptoms recur after discontinuation, which is as high as $50 \%{ }^{1,2}$ In refractory cases, videoassisted thoracic surgery (VATS) is performed for definitive diagnosis and surgical treatment, which may include lung resection and/or diaphragmatic plication. ${ }^{1}$

\section{Case Report}

The patient is a 44-year-old G1P1001 Caucasian female with CF, with a forced expiratory volume in 1 second to forced vital capacity ratio $\left(\mathrm{FEV}_{1} / \mathrm{FVC}\right)$ of $75-80 \%$. Her other past medical history includes insulin dependent pancreatic insufficiency, sinusitis and history of pneumothoraxes as a child; known intraabdominal endometriosis, reflux, hearing loss, and a body mass index of 21 . Menarche was at the age of 12 , and she had regular monthly menses, accompanied by significant pelvic pain due to her known intra-abdominal endometriosis. Patient was diagnosed with TES by clinical symptoms (no imaging was performed) and treated with a GnRH analogue for 6 months, with complete resolution of symptoms, however, return of symptoms soon after therapy cessation. After symptom recurrence, she underwent a hysterectomy and bilateral salpingo-oophorectomy, to both eliminate estrogen production by the ovaries and address her pelvic pain from intra-abdominal endometriosis. The patient was not started on any hormone replacement therapy after surgery because of concern of re-stimulating her TES. The patient had complete remission of her symptoms post-surgery, however, four and a half years later returned with monthly hemoptysis. A computed tomography scan of the chest/abdomen/pelvis showed no signs of residual ovarian tissue or endometriosis lesions, and no lesions in the lung, aside from bronchiectasis. She also had a high follicle stimulating hormone level $(120.1 \mathrm{U} / \mathrm{mL})$ and a low estradiol level (19 pg/mL), which was con-
Correspondence: Olga T. Filippova, Albany Medical Center, 2401 Harmony Mills Lofts, Cohoes, NY 12047, USA.

Tel: +1.845.661.5716. Fax: +1.518.262.2675.

E-mail: filippo@mail.amc.edu

Key words: Thoracic endometriosis; Aromatase inhibitor; Cystic fibrosis.

Contributions: all authors contributed evenly to the manuscript, in both concept and writing.

Conflict of interest: the authors declare no potential conflict of interest.

Received for publication: 17 February 2016.

Revision received: 26 July 2016.

Accepted for publication: 2 August 2016.

This work is licensed under a Creative Commons Attribution NonCommercial 4.0 License (CC BYNC 4.0).

(C) Copyright O.T. Filippova et al., 2016

Licensee PAGEPress, Italy

Chest Disease Reports 2016; 4:5815

doi:10.4081/cdr.2016.5815

sistent with the finding of no residual ovarian tissue. Because of these findings, it was decided that the patient's symptoms are most likely related to peripheral estrogen production, and she was started on an aromatase inhibitor (AI) - anastrozole $1 \mathrm{mg}$ daily by mouth, the agent used in prior studies of AI therapy on endometriosis. ${ }^{5,6}$ Six months after the initiation of therapy, the patient reports resolution of her monthly hemoptysis, and she has had no return of symptoms after almost three years of AI therapy. Because of concern of bone loss with CF and long term AI therapy, the patient is being monitored with dual-energy X-ray absorptiometry (DEXA) every two years.

\section{Discussion}

Multiple mechanisms of ascent of endometriosis lesions into the thoracic cavity have been proposed. The celomic metaplasia theory involves pathologic stimuli inducing precursor cells with the same embryologic origin in the pleura to differentiate into endometrial cells. This theory does not account for intrapulmonary endometriosis or right-sided predominance of disease. Another mechanism is retrograde menstruation with transperitoneal-transdiaphragmatic migration of endometrial tissue. This mechanism links TES to catamenial pneumothorax because during menstruating, there is an open communication between the atmosphere and the peritoneal cavity (through the lost cervical mucus 
plug and fallopian tubes), which may allow air to migrate into the peritoneal cavity, and then into the thoracic cavity through diaphragmatic fenestrations (congenital or acquired), leading to lung collapse. ${ }^{1,2}$ This theory is supported by the findings that plication of diaphragmatic perforations and tubal ligation have cured cases of catamenial pneumothorax. ${ }^{2}$ It is advised to search for endometriosis in women presenting with spontaneous diaphragmatic rupture. A third theory discusses metastatic spread of endometriosis through entry into the uterine vein. ${ }^{1}$ Women with TES often have a history of uterine manipulation or trauma (most commonly hysteroscopy or dilation and curettage), giving support to the lymphovascular embolization theory. ${ }^{1}$

Extra-ovarian estrogen production is not under hormonal control of the hypothalamicpituitary-gonadal axis, thus treatments which induce hypogonadotropic hypogonadism have little effect on extra-ovarian estrogen production. ${ }^{5}$ Aromatase is a key enzyme in all estrogen synthesis, helping convert androstenedione and testosterone into estrone and estradiol, respectively, in a rate limiting step..$^{5,7,8}$ It is known that aromatase is expressed in high levels in endometriosis implants $s^{5,7,8}$ and has been suggested that endometriosis lesions over-express aromatase, ${ }^{6,8}$ thus securing their own estrogen supply, which is in turn suppressed by AI therapy. Takayama and colleagues were the first to report successfully treating refractory endometriosis after a hysterectomy and bilateral salpingo-oophorectomy in a postmenopausal woman with an $\mathrm{AI}$ in $1998 .{ }^{9}$ In that report, the authors show not only a $50 \%$ decrease in the level of estradiol after 6 months of therapy, but essential absence of aromatase mRNA in the endometriosis implant, biopsied 6 months after starting AI treatment. $^{9}$

There are two types of AIs: type I, non-competitive, and type II, competitive, both work by binding aromatase and causing cell apoptosis. ${ }^{5,8}$ Anastrozole and letrozole are third generation type II inhibitors, and are much more specific for the aromatase enzyme. ${ }^{5,8}$ Anastrozole has been shown to relieve pain, cause lesion regression, and reduce circulating estradiol levels when used to treat pelvic endometriotis. ${ }^{5}$ One randomized controlled trial showed that using AIs with GnRH analogues significantly improved pain scores compares to GnRH analogues alone, as both ovarian and extra-ovarian estrogen production was inhibited. ${ }^{7}$ Another study showed that a GnRH analogue combined with anastrozole lengthened the pain free interval (35 vs 7.5\% recurrence rate in the first 24 months). ${ }^{6}$

Common side effects of AI therapy include headache, nausea, and diarrhea, but compared to GnRH agonists, hot flashes are milder and not as frequent. ${ }^{8}$ Because patients with CF are already predisposed to osteoporosis (because of several mechanisms - malabsorption, recurrent infections with inflammation, vitamin D and $\mathrm{K}$ deficiency, and possible direct effect of the CF mutation), ${ }^{10}$ combined with the effects of AIs on bone metabolism and risk of osteoporosis with long term use, ${ }^{8}$ this patient is being monitored with DEXA scans every two years and may require bisphosphonate therapy in the future.

There is another complicating factor in this patient, however. Do her CF and TES interact? Estradiol increases the severity of pneumonia in adult CF mice, via 2 potential mechanisms enhancement of Th17 regulated inflammation and the suppression of innate antibacterial defenses, and leads to an increase in the inflammatory/neutrophil infiltrates, mucin production, and bacterial load. ${ }^{11}$ Estradiol also has been shown to inhibit interleukin-8 release, suggesting an anti-inflammatory mechanism for E2, thus predisposing women with $\mathrm{CF}$ to infection and colonization. ${ }^{12}$ From this, it seems that controlling her TES may in fact decrease the damaging lung effects of estradiol.

\section{Conclusions}

Since this patient is afflicted with CF, any benefit that could be gained from lung surgery must be weighed against worsening her lung function, and affecting the possibility of a lung transplant in the future. Her lung function is only mildly decreased with an $\mathrm{FEV}_{1} / \mathrm{FVC}$ of 75 $80 \%$. Currently, her symptoms are controlled on AI therapy. However, if AI therapy is discontinued given the concerns of long-term AI use and her monthly hemoptysis returns, a VATS procedure may not be unreasonable to determine if TES lesions are present and/or diaphragmatic defects are visible. The presence or absence of either may help determine if lung resection has potential of helping with her symptoms (if no visible lesions are present, there is questionable benefit of blind lung resection), or if a diaphragmatic plication is necessary.

\section{References}

1. Veeraswamy A, Lewis M, Mann A, et al. Extragenital endometriosis. Clin Obstet Gynecol 2010;53:449-66.

2. Augoulea A, Lambrinoudaki I, Christodoulakos G. Thoracic endometriosis syndrome. Respiration 2008;75:113-9.

3. Maurer ER, Schaal JA, Mendez FL Jr. Chronic recurring spontaneous pneumothorax due to endometriosis of the diaphragm. J Am Med Assoc 1958;168: 2013-4.

4. Parker CM, Nolan R, Lougheed MD. Catamenial hemoptysis and pneunothoraces in a patient with cystic fibrosis. Can Respir J Vol 2007;14:295-7.

5. Simsa P, Mihalyi A, Kyama CM, et al. Selective estrogen-receptor modulators and aromatase inhibitors: promising new medical therapies for endometriosis? Women Health 2007;3:617-28.

6. Ebert AD, Bartley J, David M. Aromatase inhibitors and cyclooxygenase-2 inhibitors in endometriosis: new questions - old answers? Eur J Obstet Gyn R B 2005;122: 144-50.

7. Patwardhan S, Nawathe A, Yeates D, et al. Systematic review of the effects of aromatase inhibitors on pain associated with endometriosis. Int J Gynecol Obstet 2008; 115:818-22.

8. Attar E, Bulun SE. Aromatase inhibitors: the next generation of therapeutics for endometriosis? Fertil Steril 2006;85:130718.

9. Takayama K, Zeitoun K, Guncy RT, et al. Treatment of severe postmenopausal endometriosis with an aromatase inhibitor. Fertil Steril 1998;69:709-13.

10. Javier RM, Jacquot J. Bone disease in cystic fibrosis: what's new? Joint Bone Spine 2011;78:445-50.

11. Wang Y, Cela E, Gagnon S, Sweezey NB. Estrogen aggravates inflammation in Pseudomonas aeruginosa pneumonia in cystic fibrosis mice. Resp Res 2010;11:16678.

12. Chotirmall SH, Greene CM, Oglesby IK, et al. 17 $\beta$-estradiol inhibits IL-8 in cystic fibrosis by up-regulating secretory leucoprotease inhibitor. Am J Resp Crit Care 2010;182:62-72. 